Supporting information

\title{
Super tough PLA plasticized with epoxidized soybean oil methyl ester for flexible food packaging.
}

Arkadiusz Zych*, Giovanni Perotto, Dagmara Trojanowska, Giacomo Tedeschi, Laura

Bertolacci, Nora Francini and Athanassia Athanassiou*

\section{Corresponding Author}

*Arkadiusz Zych, e-mail: Arkadiusz.Zych@iiit.it

*Athanassia Athanassiou, e-mail: Athanassia.Athanassiou@iit.it

ESOME (epoxidized soybean oil methyl ester)
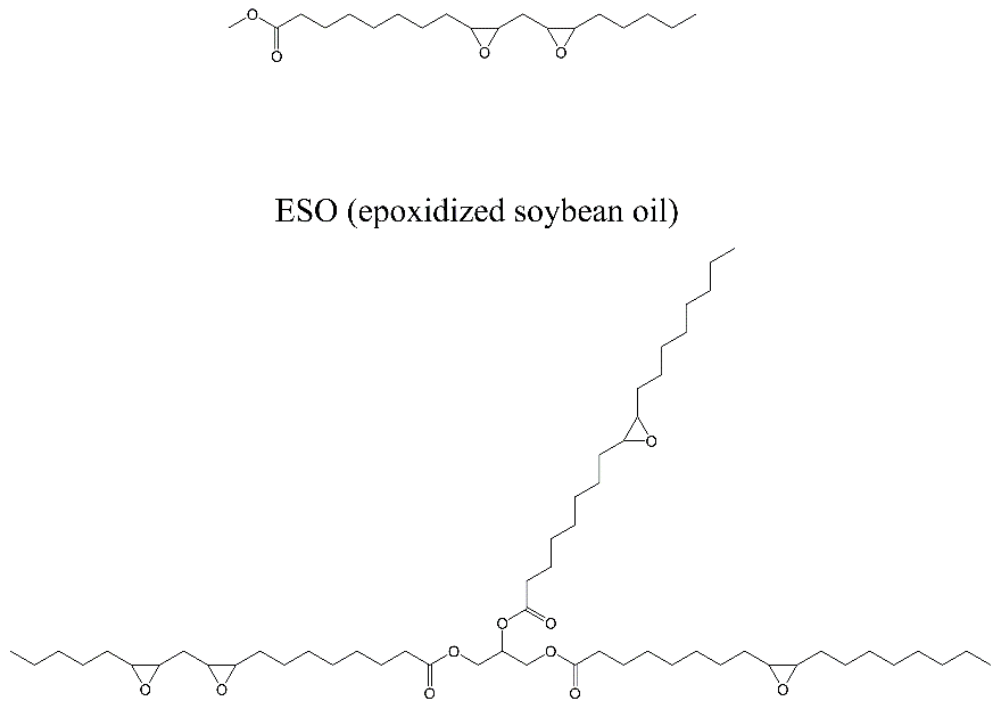

Figure S1. Comparison of the ESOME and ESO structures. 


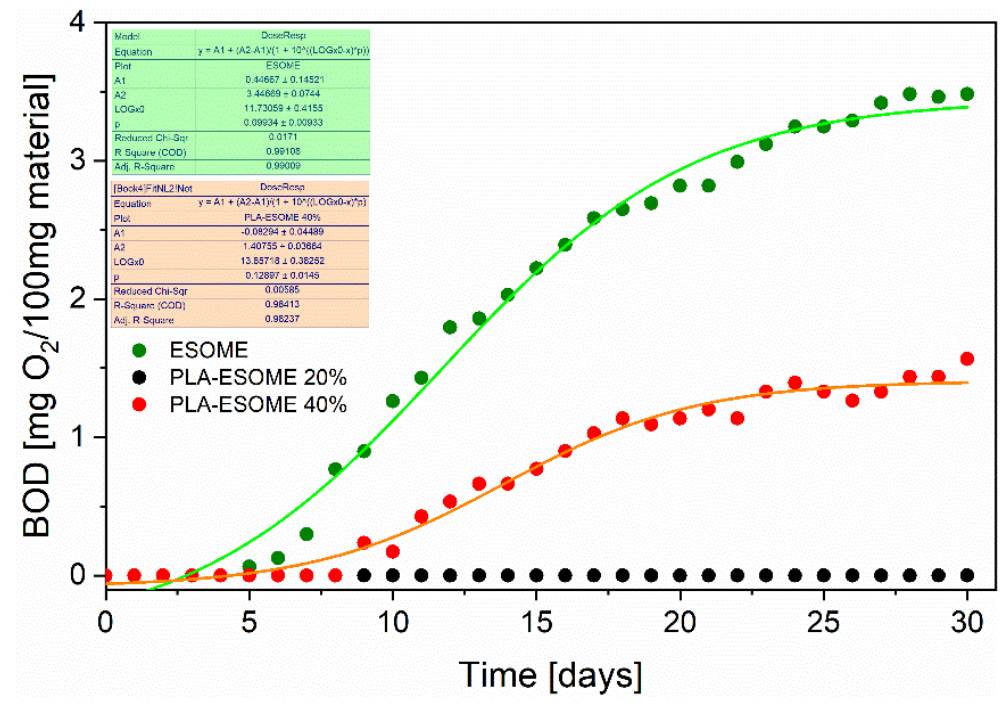

Figure S2. Biochemical oxygen demand (BOD) of ESOME, PLA-ESOME 20\% and PLA-ESOME $40 \%$.

The experimental points were fitted with a sigmoidal growth equation, obtaining a good correlation. The analysis allowed to extrapolate the value of BOD expected at the plateau (parameter A2 in the equations), which is in agreement with the fact that the curves almost reached the plateau. These values allows to calculate the ratio between BOD of the PLA-ESOME 40\% sample and BOD of the pure ESOME at the plateau.

Table S1. Analysis of the extrapolated maximum BOD.

\begin{tabular}{ccc}
\hline sample & $\begin{array}{c}\text { BOD at plateau } \\
{\left[\mathrm{mg} \mathrm{O}_{2} / 100 \text { mg material }\right]}\end{array}$ & $\begin{array}{c}\text { BOD } \\
\text { ESOmposite/ BOD ESOME } \\
\text { at plateau }\end{array}$ \\
\hline PLA-ESOME $40 \%$ & $3.45($ error $2.2 \%)$ & - \\
PLA-ESOME 20\% & $1.41($ error $2.6 \%)$ & $40.8 \%$ \\
\hline
\end{tabular}

These results suggest that only the ESOME fraction (40\%) underwent biodegradation in the PLAESOME 40\% material. Signals for PLA-ESOME 20\% sample were too low to be detected. 


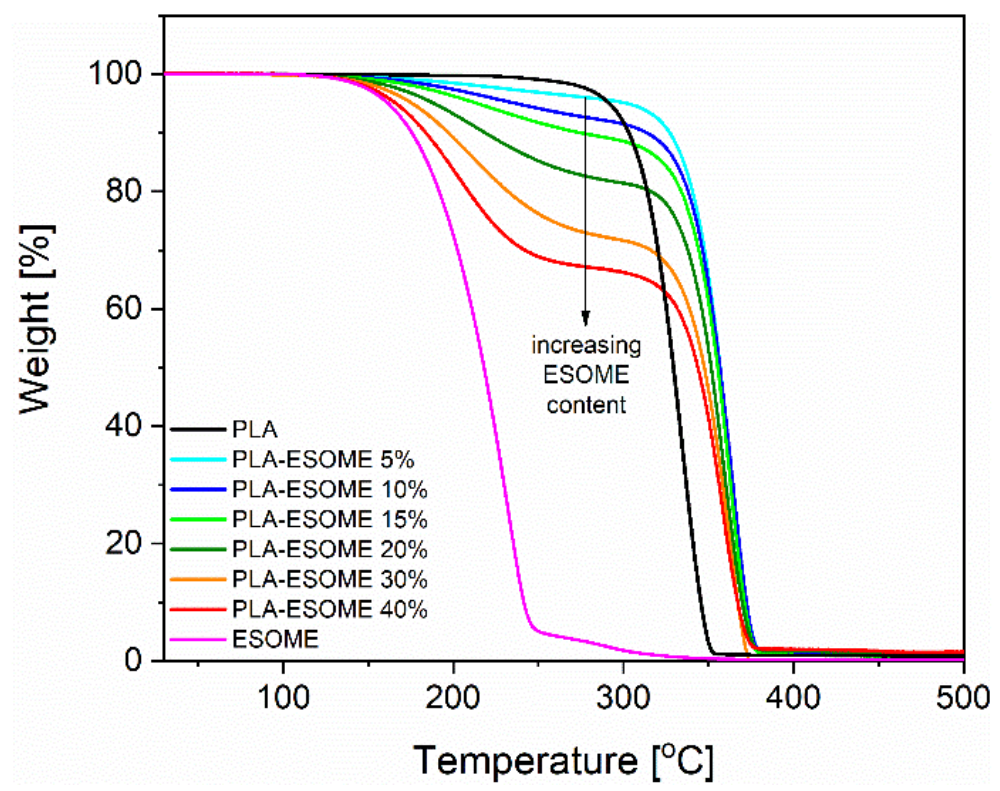

Figure S3. TGA curves of PLA, ESOME and PLA-ESOME samples.

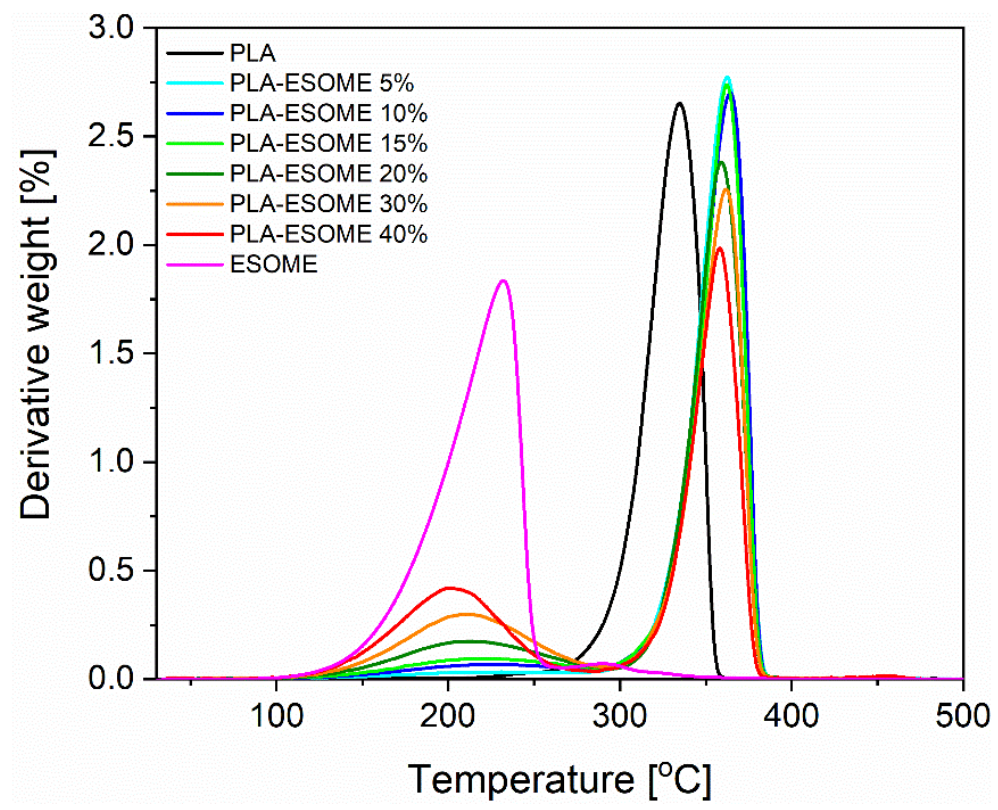

Figure S4. DTGA curves of PLA, ESOME and PLA-ESOME samples. 
Table S2. Onsets and peak maxima determined from the TGA and DTGA curves.

\begin{tabular}{ccccc}
\hline & $\begin{array}{c}\text { max } \\
{\left[{ }^{\circ} \mathrm{C}\right]}\end{array}$ & $\begin{array}{c}\text { max } \\
\text { derivative } 1 \\
{\left[{ }^{\circ} \mathrm{C}\right]}\end{array}$ & $\begin{array}{c}\text { onset } 2 \\
{\left[{ }^{\circ} \mathrm{C}\right]}\end{array}$ & $\begin{array}{c}\text { merivative 2 } \\
{\left[{ }^{\circ} \mathrm{C}\right]}\end{array}$ \\
\hline PLA & - & - & 311.05 & 332.05 \\
PLA ESOME 5\% & 157.6 & 215.72 & 341.18 & 362.36 \\
PLA ESOME 10\% & 165.32 & 218.98 & 342.56 & 364.21 \\
PLA ESOME 15\% & 162.45 & 214.58 & 342.81 & 362.07 \\
PLA ESOME 20\% & 162.18 & 207.95 & 339.84 & 358.96 \\
PLA ESOME 30\% & 164.51 & 210.33 & 341.24 & 362.08 \\
PLA ESOME 40\% & 160.97 & 201.74 & 339.14 & 358.08 \\
ESOME & 191.75 & 232.06 & - & - \\
\hline
\end{tabular}

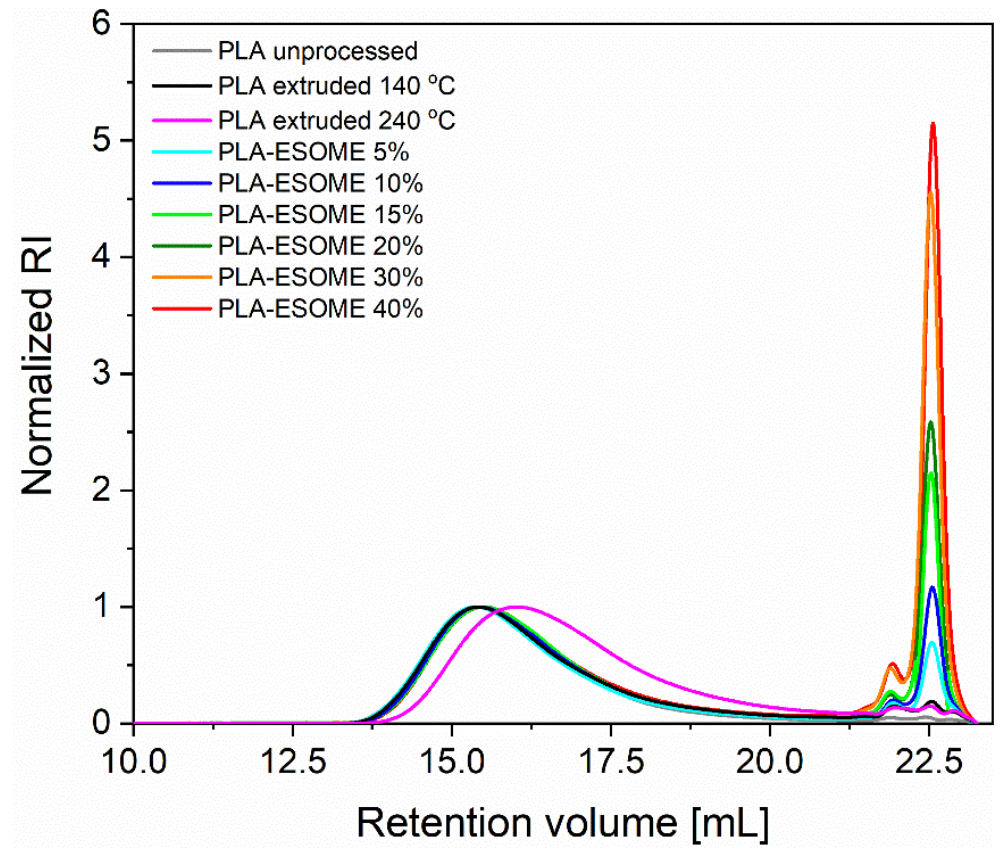

Figure S5. GPC chromatograms of PLA and PLA-ESOME samples. 
Table S3 . Molecular weights and dispersities of PLA and PLA-ESOME samples determined by GPC.

\begin{tabular}{cccc}
\hline Sample & $\begin{array}{c}\mathrm{M}_{\mathrm{n}} \\
(\mathrm{kg} / \mathrm{mol})\end{array}$ & $\begin{array}{c}\mathrm{M}_{\mathrm{w}} \\
(\mathrm{kg} / \mathrm{mol})\end{array}$ & $Ð$ \\
\hline PLA unprocessed & 103 & 138 & 1.34 \\
PLA extruded $140^{\circ} \mathrm{C}$ & 96 & 126 & 1.32 \\
PLA extruded $240^{\circ} \mathrm{C}$ & 67 & 93 & 1.40 \\
PLA ESOME 5\% & 102 & 132 & 1.29 \\
PLA ESOME 10\% & 102 & 135 & 1.32 \\
PLA ESOME 15\% & 103 & 133 & 1.30 \\
PLA ESOME 20\% & 103 & 135 & 1.31 \\
PLA ESOME 30\% & 102 & 132 & 1.29 \\
PLA ESOME 40\% & 103 & 132 & 1.28 \\
\hline
\end{tabular}

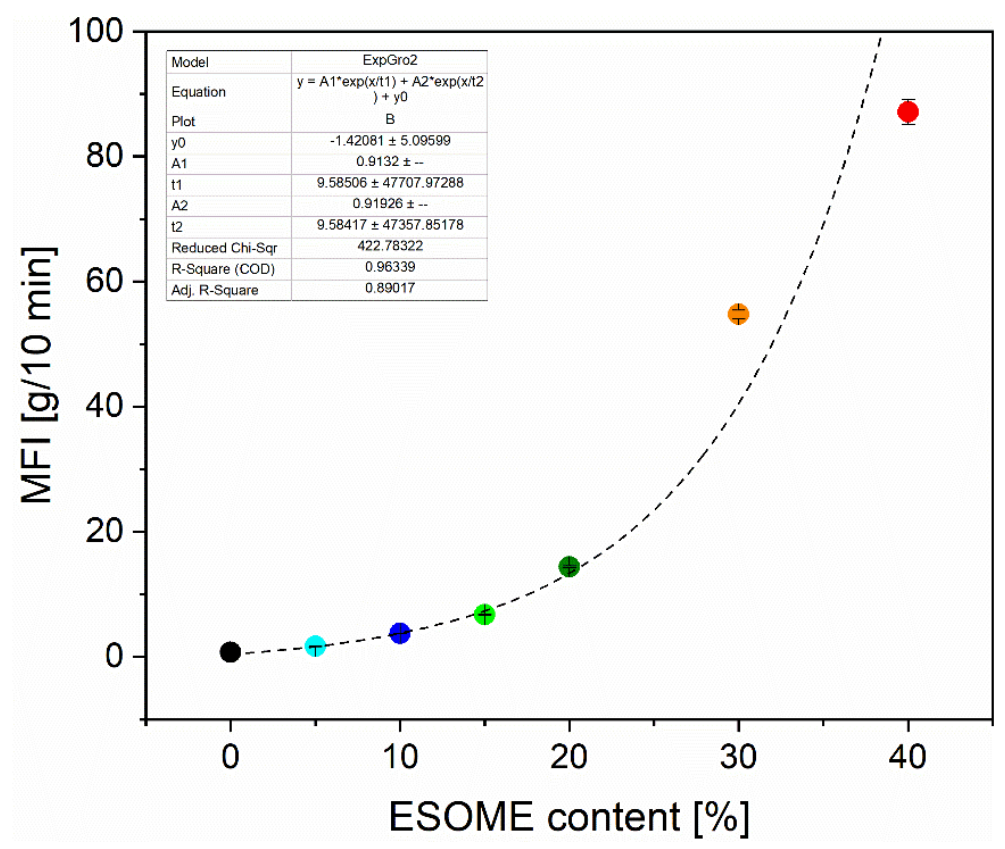

Figure S6. Exponential growth of melt flow index (MFI) with increasing ESOME content. 
A

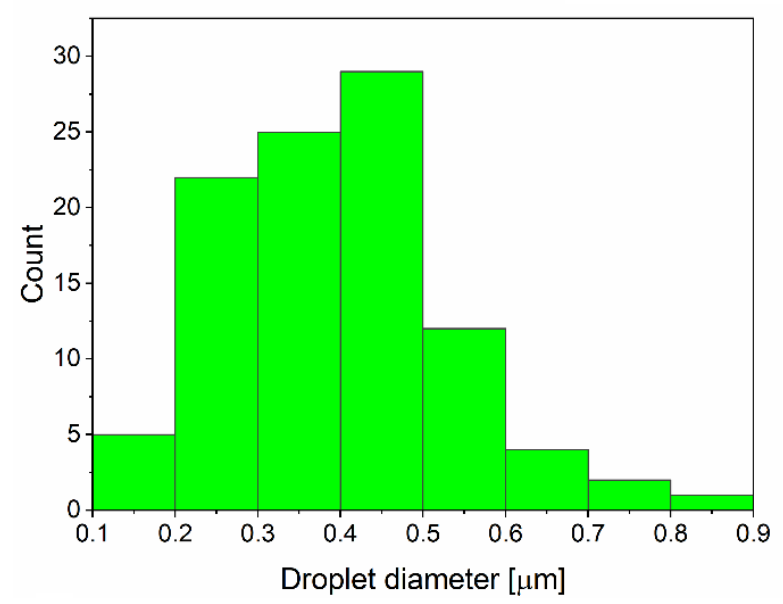

C

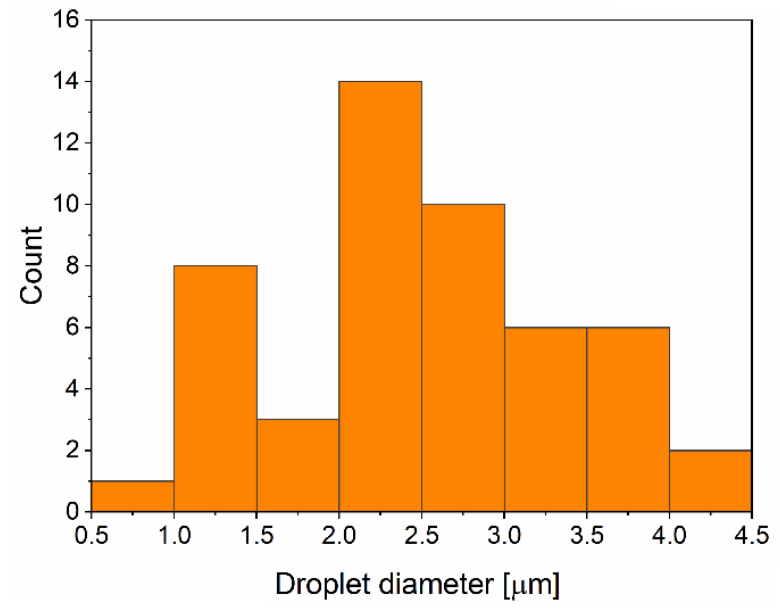

B

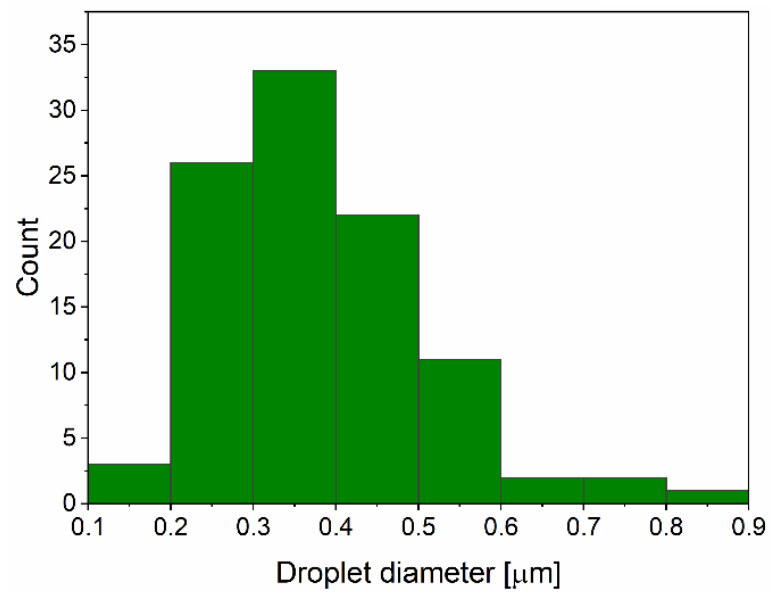

D

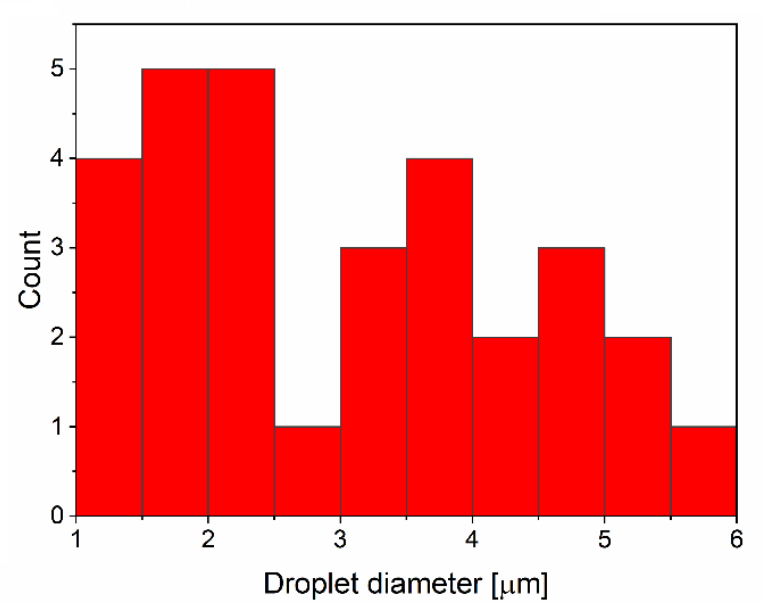

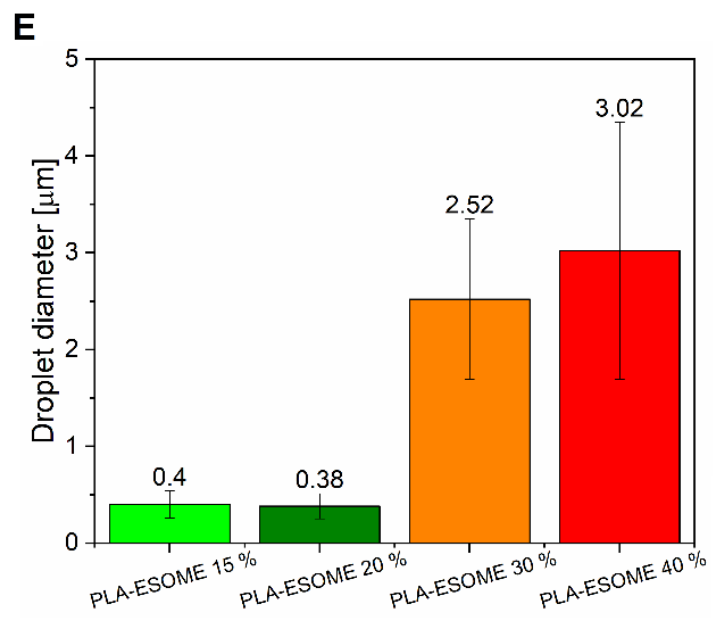

Figure S7. ESOME droplet size distribution from SEM images: (A) PLA-ESOME 15\%, (B) PLAESOME 20\%, (C) PLA-ESOME 30\%, (D) PLA-ESOME 40\%, (E) average values with error bars. 
Table S4. Mean and standard deviation of droplets diameter for PLA-ESOME samples.

\begin{tabular}{ccc}
\hline Sample & Mean & $\begin{array}{c}\text { Standard } \\
\text { deviation }\end{array}$ \\
\hline PLA-ESOME 15\% & 0.40 & 0.14 \\
PLA-ESOME 20\% & 0.38 & 0.13 \\
PLA-ESOME 30\% & 2.52 & 0.83 \\
PLA-ESOME 40\% & 3.01 & 1.32 \\
\hline
\end{tabular}

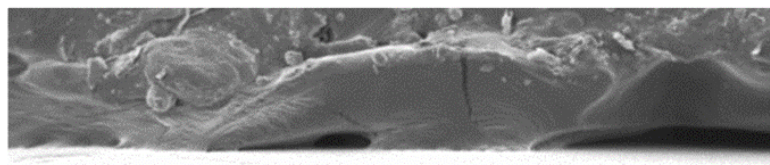

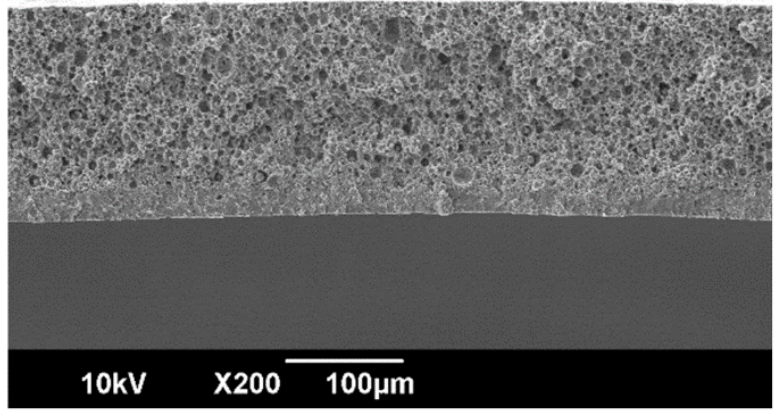

PLA-SOME 40\%

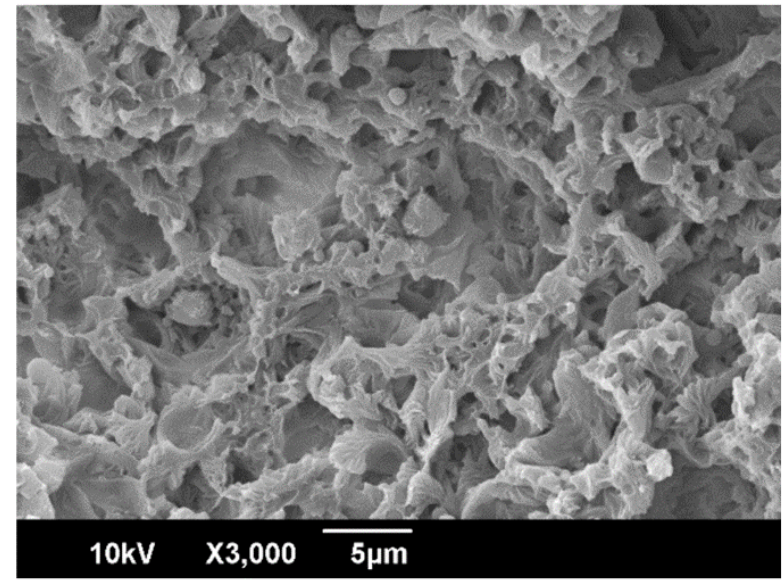

PLA-ESOME 40\%

Figure S8. Freeze-fractured surface SEM images of PLA-ESOME 40\% after removal of ESOME in $95 \%$ ethanol for $2 \mathrm{~h}$. 


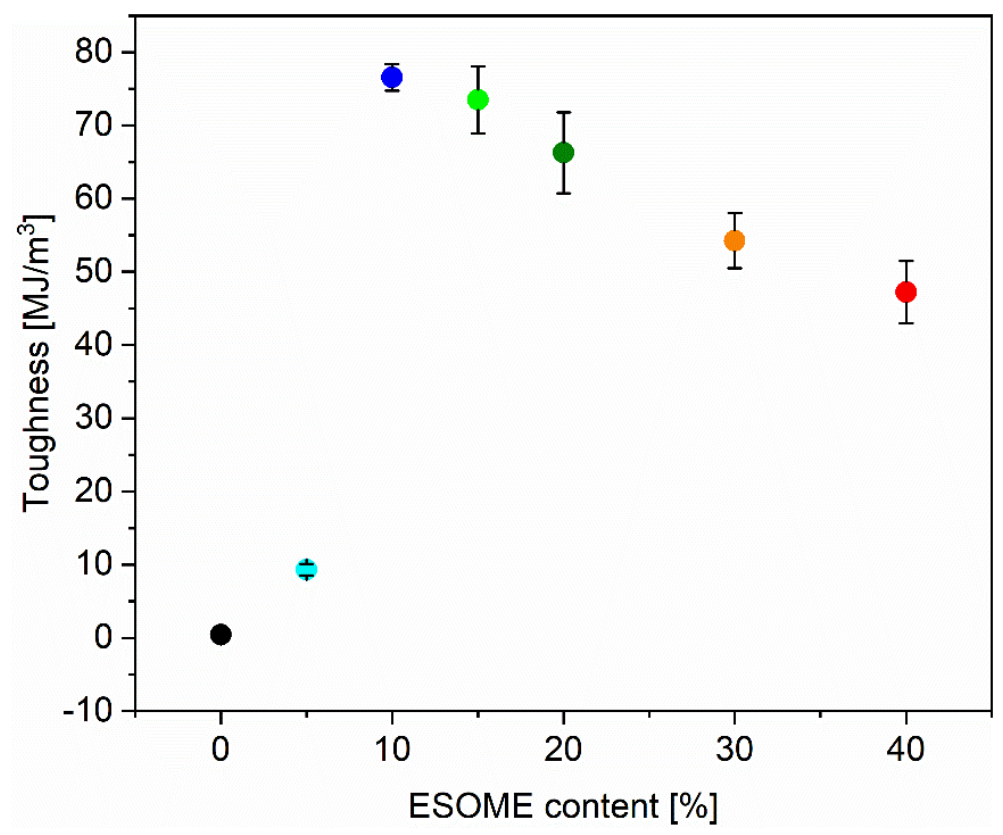

Figure S9. Relationship between toughness and ESOME content.

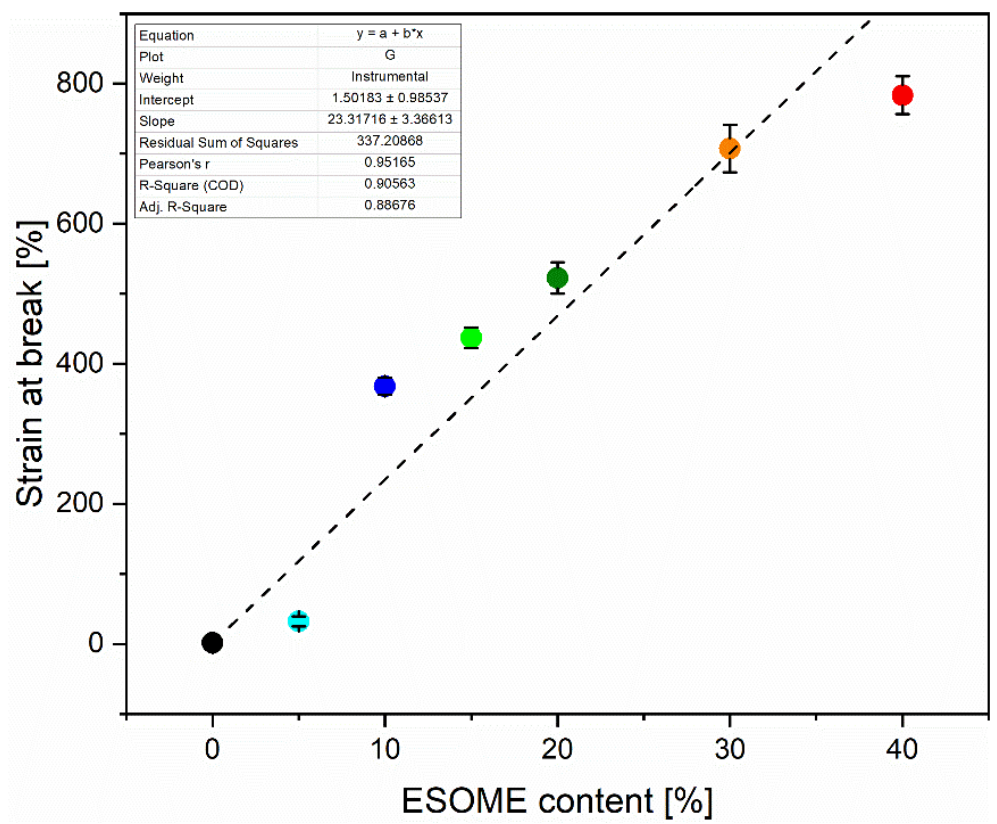

Figure S10. Linear increase of strain at break with increasing ESOME content. 


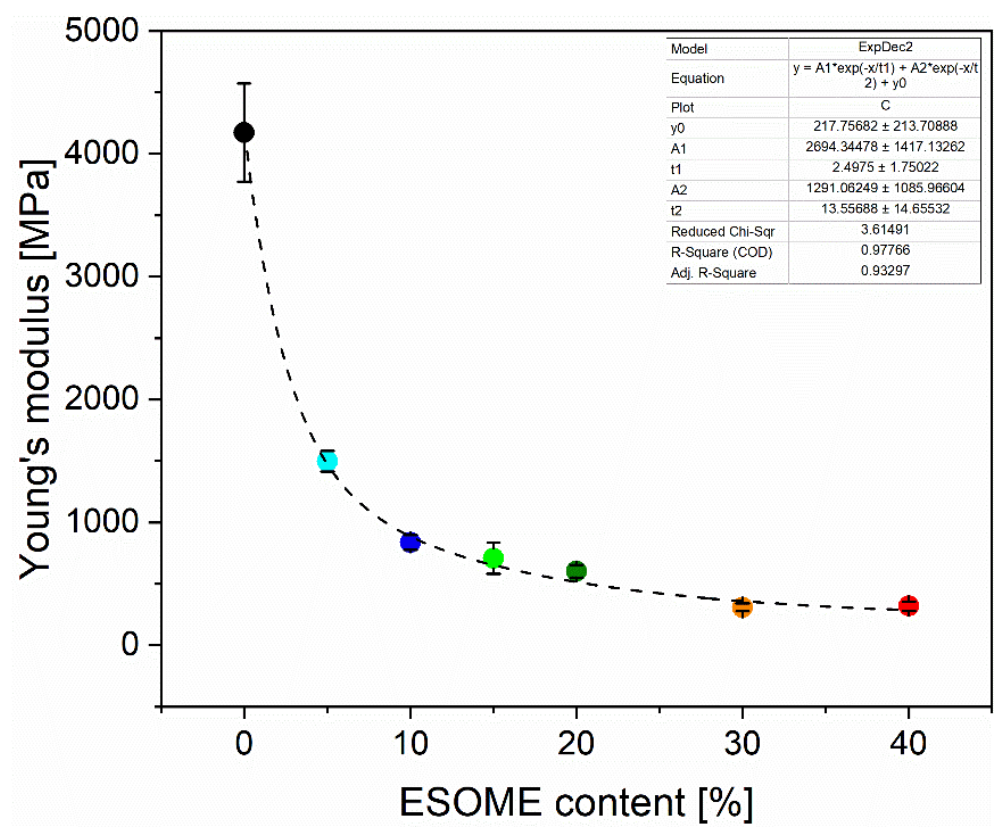

Figure S11. Exponential decay of Young's modulus with increasing ESOME content.

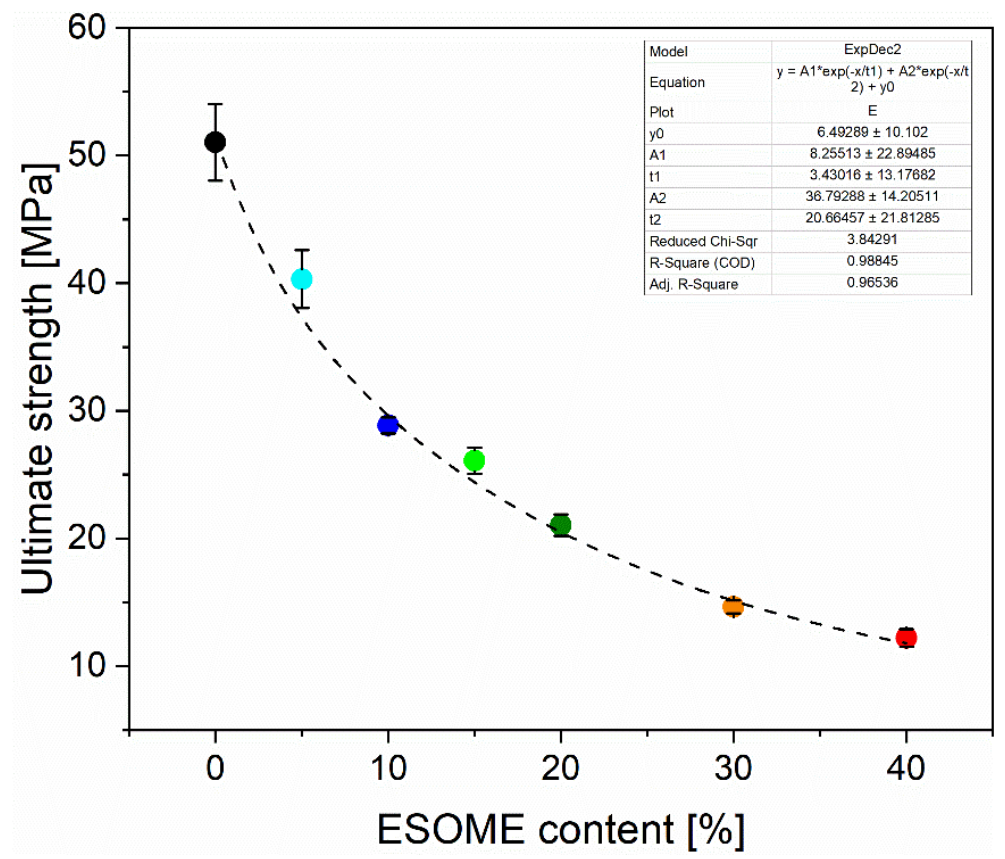

Figure S12. Exponential decay of ultimate strength with increasing ESOME content. 


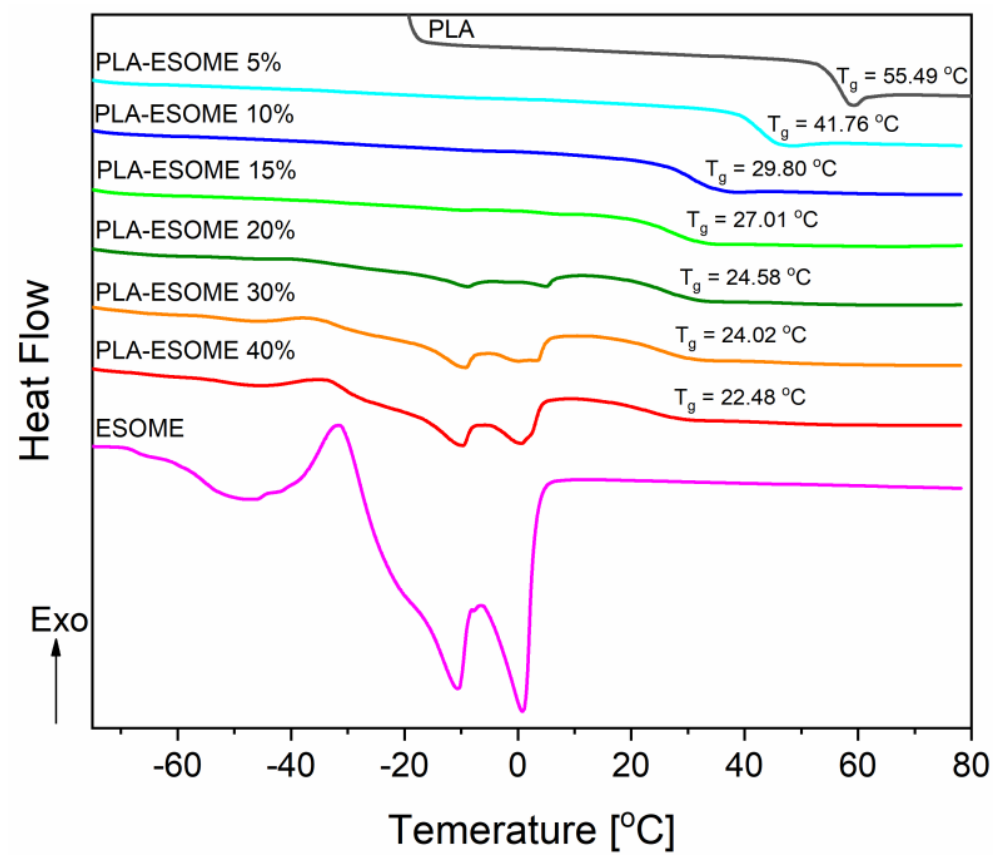

Figure S13. DSC curves of PLA, PLA-ESOME samples and ESOME.

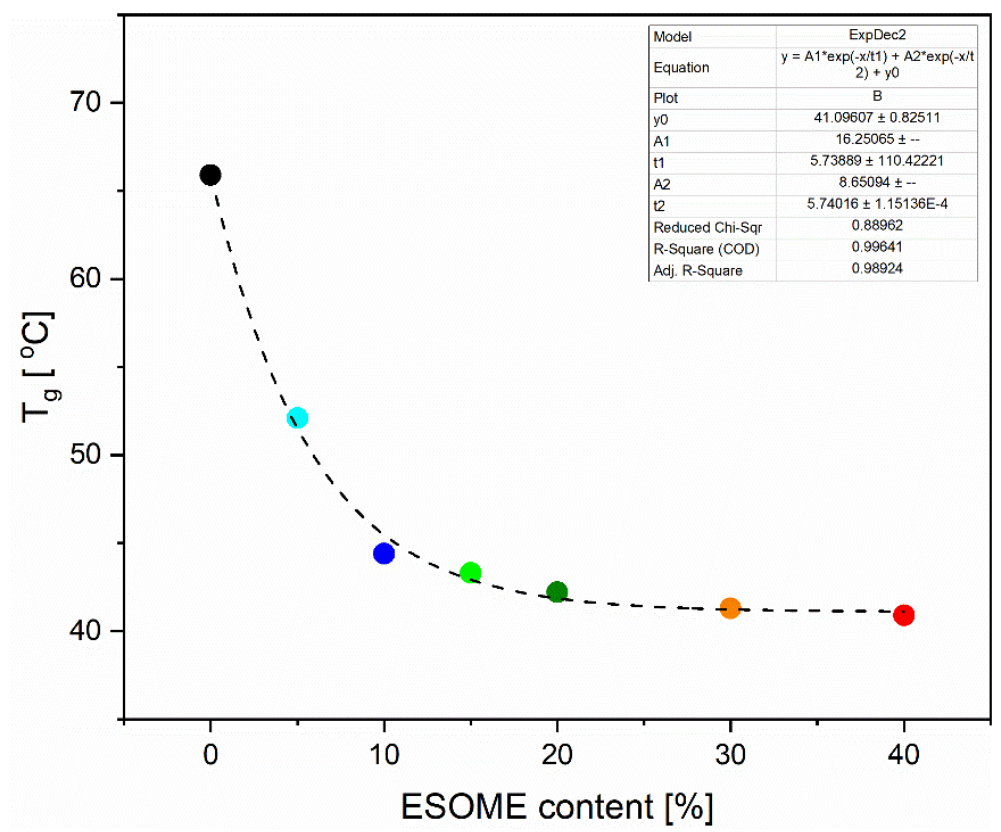

Figure S14. Exponential decay of the glass transition temperature $\left(\mathrm{T}_{\mathrm{g}}\right)$ with increasing ESOME content. 


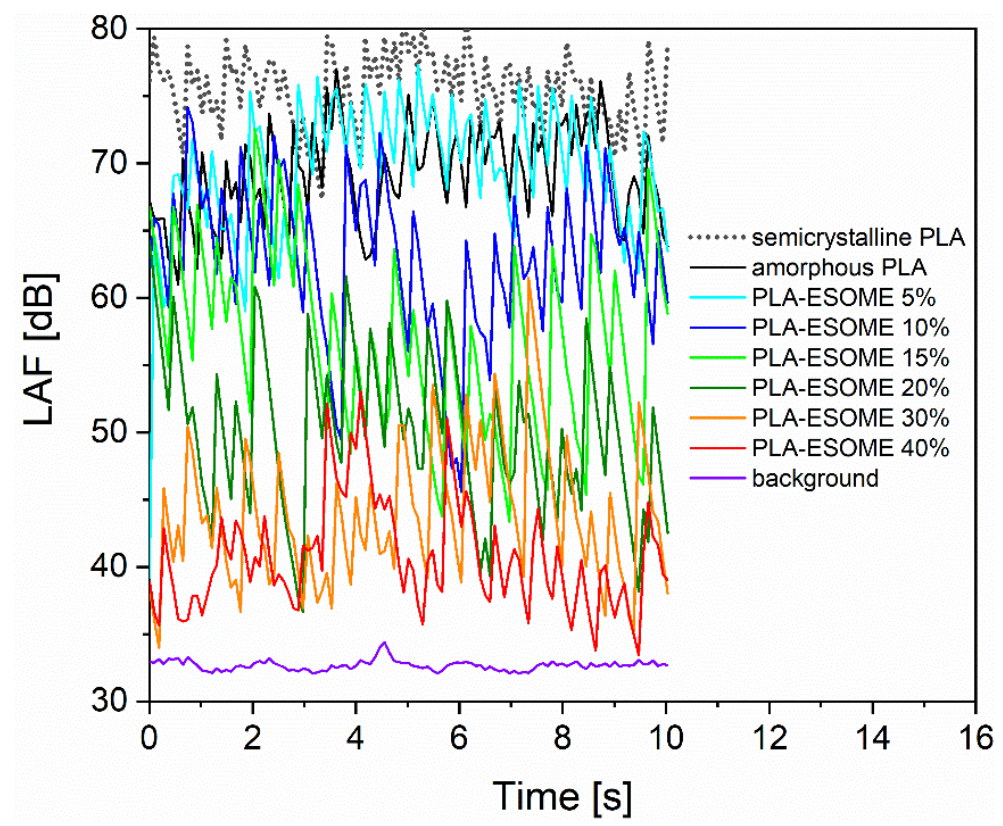

Figure S15. Sound levels with A type frequency weighting and fast time weighting of $125 \mathrm{~ms}$ (LAF) of PLA and PLA-ESOME samples.

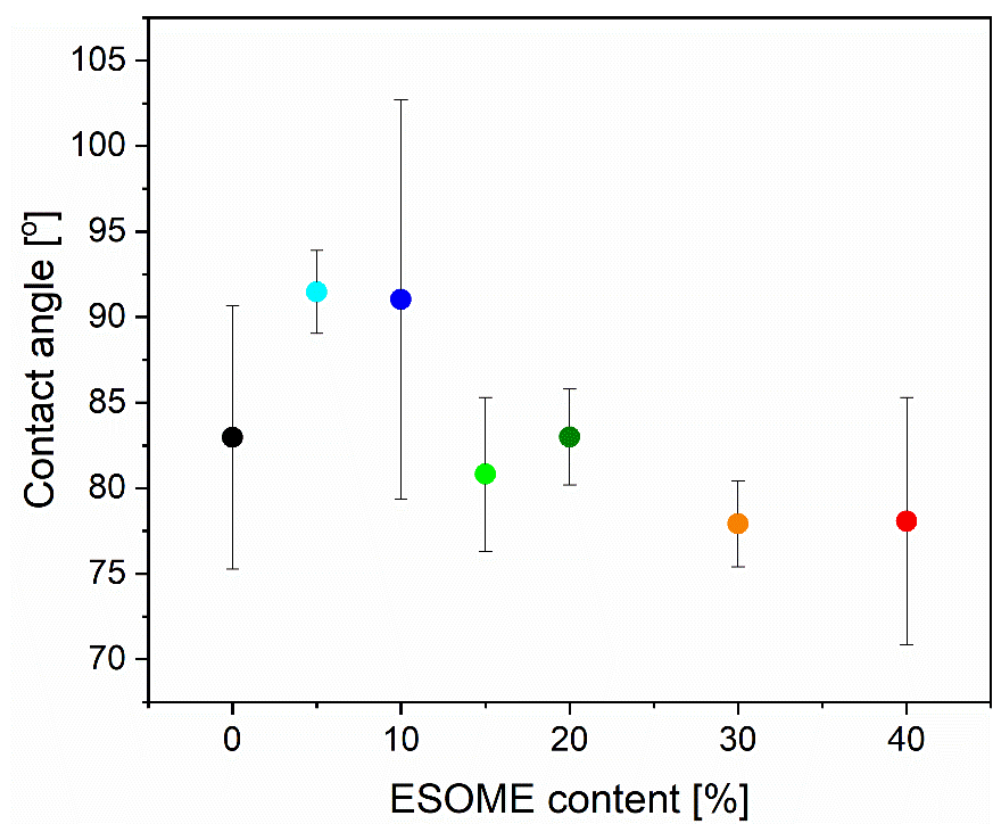

Figure S16. Water contact angle (WCA) of PLA and PLA-ESOME samples. 